\title{
The data and safety monitoring board in sponsored pediatric clinical trials: practical applications
}

\author{
Pramod M. Lad, Rebecca Dahl \\ Human Subjects Protection Program, Children's Hospital, Los Angeles, United States
}

Received: October 8, 2014

Accepted: December 9, 2014 Online Published: January 13, 2015

DOI: $10.5430 /$ jha.v4n1p57

URL: http://dx.doi.org/10.5430/jha.v4n1p57

\begin{abstract}
The Data and Safety Monitoring Board (DSMB) monitors the progress of clinical trials for safety and implements stopping rules as needed. Although NIH and FDA guidelines recommend the use of a DSMB for phase I, II, and III pediatric clinical trials, the manner in which the DSMB is constituted has received little attention. In this study we reviewed the Institutional Review Board (IRB) applications submitted between 2008 and 2012 at our institution (Children's Hospital Los Angeles) for phase I, II and III studies which were multi-site, sponsored and performed under a sponsor's Investigation New Drug Application (IND) for the type of data and safety monitoring that was being used. Our results indicate that approximately two-third of the studies used an independent DSMB, 10\% utilized a sponsor's DSMB and the remaining studies (25\%) did not utilize a DSMB and relied instead on safety monitoring by the Principal Investigator (PI) and the medical monitor/director. This pattern was observed across all study phases and for blinded as well as unblinded studies. Our result suggests that a Data and Safety Monitoring Plan (DSMP), although required by the IRB, is rarely submitted by the sponsor at the time of application. Instead the DSMP is submitted to the IRB by the PI on IRB supplied templates. IRB review of these completed templates were critical to ensuring DSMB related compliance. Additionally, a significant percent of sponsored clinical trials used the PI or an individual designated as medical director/monitor, rather than constituting a DSMB.
\end{abstract}

Key Words: Institutional review board, Good clinical practice, Quality assurance, Data and safety monitoring board

\section{Background}

\subsection{Data and safety monitoring board}

Data and safety monitoring boards were proposed as far back as 1967 when the Greenberg report from the National Heart Institute proposed that clinical trial data should be collected and analyzed to monitor the progress of a clinical trial. Since then, Data and Safety Monitoring Board (DSMB) have become a standard part of clinical trial practice, ${ }^{[1]}$ even though some authors have questioned whether all clinical trials should have a DSMB. ${ }^{[2]}$

The main functions of data and safety monitoring boards are summarized below:

Main functions of the DSMB:

- Monitor the conduct and progress of the clinical study

- Assess harms and benefits to research subjects

- Protect the safety of human subjects

- Determine whether the clinical trial should continue with change, continue as planned or be stopped

Minor functions of the DSMB:

- Assess need for changes in protocol in particular procedures or eligibility

\footnotetext{
*Correspondence: Pramod M. Lad; Email: plad@chla.usc.edu; Address: Children’s Hospital Los Angeles, 4650 Sunset Boulevard, Mailstop \#23, CA 90027, United States.
} 
- Monitor efficiency of enrollment and data collection

- Monitor other aspects of clinical trial conduct including protocol deviations as charter suggests

Among the major functions of the board are the assessments of potential harms/risks and benefits to ensure that the safety of human subjects is protected (Good Clinical Practice Guidelines). This is largely done through the analysis of serious adverse events reported to the DSMB during the course of the study. The collection and submission of adverse event report is performed by the sponsor of the clinical trial who has in turn gathered this information from participating sites. ${ }^{[3]}$ After a review of these events the DSMB issues a report which recommends continuance with or without changes in the study protocol. An important procedure for the DSMB is the application of the stopping rules which indicate that the progress of the clinical trial be stopped either because harm or benefit is statistically proven. ${ }^{[4]}$ Another important procedure is the recommendation of a change in the course of the study (e.g. reassessment of including a particular study arm or changes in the inclusion or exclusion criteria) to protect future subjects.

The DSMB typically consists of at least three members-two physicians in the therapeutic area and one biostatistician. In practice, DSMBs tend to have larger membership. A DSMB is sometimes referred to as the Data and Safety Monitoring Committee (DSMC), but there is little difference in their roles. The difference is primarily one of nomenclature. For the purpose of this article the term DSMB will also include the DSMC.

A DSMB can take several forms. ${ }^{[5]}$ It can be constituted at the local site level and consist of the PI and participating members from the site's institution, or from the sponsor's institution. A sponsor's DSMB can consist of the sponsor's staff (including the biostatistician) as well as external consultants. An independent DSMB should consist of members typically not associated with the sponsor in any way and include an independent biostatistician.

It should also be clear what does not constitute a DSMB. Assessment of serious adverse events by the PI alone at the site is not a DSMB. Also evaluation of the SAEs by the sponsor's medical monitor/medical director, with or without involvement of the PI, does not fulfill the criteria for a DSMB.

\subsection{Data and safety monitoring plan}

It is advisable that the sponsor create a data and safety management plan prior to the initiation of the clinical trial. The possible contents of the Data and safety monitoring plan (DSMP) are described based on our own institution's requirements.

Overall risk assessment:

Risk assessment grid (lists procedures by risk) rank study as minimal risk, moderate risk or significant risk. (All phase 14 studies are rated as significant risk.)

Creating the plan:

- Who will perform the safety monitoring (PI, internal DSMC or independent DSMB (sponsor)

- Who will monitor safety at the institution? Identify team members

- If internal; DSMC composition and responsibilities of members

- If independent DSMB composition and responsibilities of the members

- Safety monitoring methods and intervals

- Grading method for adverse events

- Attribution scales for adverse events

- Agencies to whom the SAEs will be reported

- Decision making criteria (stopping rules)

- Frequency of meeting of DSMB

- Interval safety assessment: specify patient number or time

- DSMB meetings and discussions: dissemination of reports to agencies

The main items include: who will monitor the study, the DSMB/DSMC composition, the monitoring methods, frequency of meetings, stopping rules, and the unit for safety assessments (event or interval based). While the regulations do not stipulate a particular format for such a plan these basic ingredients for a DSMP seem essential. It is advisable to submit the Plan to the Institutional Review Board (IRB) for review along with the protocol. Some sponsors may choose to integrate a portion of the DSMP in the protocol itself.

\subsection{NIH guidelines for DSMBs (NIH guidelines)}

The general guidelines for a DSMB are presented below:

- In addition to the DSM plan, a DSMB is generally required for Phase III clinical trials. For earlier medication trials (Phase I or II) and some behavioral clinical trials, a DSMB may be appropriate if the study has multiple clinical sites, is blinded, tests a high-risk intervention, or is conducted in vulnerable populations. Generally, Phase III trials involve larger numbers of subjects, but size is not a specific criterion for this designation.

- The purpose of the DSMB is to monitor the safety of the interventions and the validity and integrity of the data from clinical trials that require a DSMB.

- The decision to establish a DSMB is commensurate with the level of risk and/or the number of treatment sites participating in the study. A DSMB may be required by the grantee's institution, but the ultimate decision rests with the sponsoring Institute. In conducting its reviews and making recommendations, the 
DSMB works to assure that the safety of study subjects is protected while the scientific goals of the study are being met. In monitoring the data and safety of the trial, The DSMB may recommend continuation of the trial, modifications to the trial, or termination of the trial in the event of overwhelmingly significant efficacy difference between groups or unacceptable adverse events.

The NIH guidelines differ from institute to institute although general recommendations have been issued twice in the last several years. Briefly, the guidelines across major institutions suggest that a DSMP be developed for all phase 13 clinical trials. With regard to the DSMB, the guidelines suggest that all phase 3 trials have a DSMB, and that factors which may influence the decision to form a DSMB include the presence of multiple sties, blinded trials, high risk protocols and clinical trials in vulnerable subjects. Since children are considered to be vulnerable subjects, all pediatric clinical trials (phase I, II, III) should have a DSMB. The NIH guidelines do not stipulate whether an independent DSMB is required. For NIH sponsored clinical trials the sponsoring institution may have a DSMB in order to oversee multiple clinical trials.

\subsection{FDA guidelines for DSMBs (FDA guidelines)}

The FDA guidelines for a DSMB are presented below. A DSMB should be constituted if the following considerations apply.

- The study endpoint is such that a highly favorable or unfavorable result, or even a finding of futility, at an interim analysis might ethically require termination of the study before its planned completion;

- There are a priori reasons for a particular safety concern, as, for example, if the procedure for administering the treatment is particularly invasive;

- There is prior information suggesting the possibility of serious toxicity with the study treatment;

- The study is being performed in a potentially fragile population such as children, pregnant women or the very elderly, or other vulnerable populations, such as those who are terminally ill or of diminished mental capacity;

- The study is being performed in a population at elevated risk of death or other serious outcomes, even when the study objective addresses a lesser endpoint;

- The study is large, of long duration, and multi-center

The surprising aspect of the FDA guidelines is that while DSMB formation is recommended, it is not mandated for all situations. Rather the determination of whether a DSMB is constituted is left up to the sponsor. Like the NIH guidelines, the FDA guidelines (only a portion of which are included above) suggest that a DSMB be formed in multi-site, high risk, blinded trails and are strongly recommended for Phase 3 trials. Trials of short duration and small numbers of subjects (which encompass many phase 1 or phase 2 studies) may not require a DSMB. It is recommended that all clinical trials, which include children, have a DSMB. Thus for pediatric clinical trials the recommendation of the NIH and the FDA overlap.

\subsection{Role of the IRB and the IRB/DSMB interface}

The role of the IRB in safety assessment and the IRB/DSMB interface has been analyzed in the literature. ${ }^{[6]}$ The IRB is required to carry out safety assessment in the form of a review of serious adverse events, and protocol deviations (Code of Federal regulation 21 CFR Part 56). The key difference between the safety review of the IRB and the DSMB is that the IRB primarily reviews serious adverse events at the local site. It is not designed to evaluate multi-site safety events, and does not have the capability to break blinds in the evaluation of blinded clinical trials. In addition, the composition of the IRB differs markedly from that of the DSMB which is generally comprised of several physicians in the specialty, and one or more biostatisticians as well as consultants from the sponsor who are experts on the protocol. ${ }^{[7]}$

The literature has repeatedly emphasized that the IRB is not a DSMB. This interface should consist of the following:

- The IRB reviews all DSMPs at the time the study application is submitted.

- The IRB ensures that DSMB reports are received in a timely fashion and may delay the approval of the application at the annual review of the study if DSMB reports have not been filed with the IRB.

- If the DSMB recommends a change to the protocol then the IRB may suspend a study until a study amendment has been filed by the PI integrating the DSMB recommendations into the protocol.

- If the DSMB has suspended a study for safety then the IRB can immediately suspend a protocol for further enrollment and or follow up as suggested by the DSMB. The suspension may not be lifted until the DSMB recommendations have been met.

\section{Methods}

The inclusion and exclusion criteria for studies to be analyzed are listed below:

Inclusion: Industry sponsored phase 1, phase 2, and phase 3 studies.

Exclusion: All Phase 4 studies; NIH sponsored clinical trials (with or without an IND); Investigator initiated IND studies.

The information collected in this manuscript is based on the following questions: 
(1) At the time of initial submission of the application to the IRB was there a DSMP from the sponsor or the PI included in the application?

(2) Was the study industry sponsored under an IND and was it a Phase I II, or III study?

(3) Was the study a multi-site study?

(4) Was the study blinded?

(5) Did the study have a local DSMB/DSMC, a sponsor based DSMB or an independent DSMB?

(6) Was the data and safety monitoring delegated to an individual (PI or sponsor's medical monitor)?

\section{Data collection methods}

The data collected to answer these questions was gathered from the following sources:

(1) The IRB application submitted for initial approval of the protocol
(2) The DSMP attached to the application

Additionally, from the IRB application the following items were noted:

(1) The industry sponsorship of the clinical trial

(2) The phase of the study

(3) Whether the study was multi-site and or blinded

(4) The study review period

The DSMP template located at the end of this article, specifically determines whether the DSMB is involved or not, and if a DSMB is involved whether it is local, sponsor based or independent.

\section{Results}

The data is summarized in Table 1.

Table 1: DSMB patterns analyzed by phase of study

\begin{tabular}{|c|c|c|c|c|}
\hline Multisite & Blind & DSMP Sponsor & DSMP IRB & DSMB \\
\hline Phase 1 & 2 (Yes) & 0 & 2 & Independent $=1 ;$ Sponsor $=1 ;$ Local $=0 ;$ PI $/$ Medical Monitor $=2$ \\
\hline Phase 1 & 18 (No) & 0 & 18 & Independent $=12 ;$ Sponsor $=0 ;$ Local $=0 ;$ PI $/$ Medical Monitor $=6$ \\
\hline Phase 1 Total & 20 & 0 & 20 & $\begin{array}{l}\text { Independent = } 13(65 \%) ; \text { Sponsor = } 1(5 \%) ; \text { Local = 0; PI/Medical } \\
\text { Monitor }=6(30 \%)\end{array}$ \\
\hline Phase 2 & 7 (Yes) & 0 & 7 & Independent $=6 ;$ Sponsor $=0 ;$ Local $=0 ;$ PI $/$ Medical Monitor $=1$ \\
\hline Phase 2 & 6 (No) & 0 & 6 & Independent $=4 ;$ Sponsor $=2 ;$ Local $=0 ;$ PI $/$ Medical Monitor $=0$ \\
\hline Phase 2 Total & 13 & 0 & 13 & $\begin{array}{l}\text { Independent = } 10(76 \%) \text {; Sponsor = } 2(15 \%) \text {; Local = 0; PI/Medical } \\
\text { Monitor }=0\end{array}$ \\
\hline Phase 3 & 16 (Yes) & 0 & 16 & Independent $=8 ;$ Sponsor $=2 ;$ Local $=0 ;$ PI $/$ Medical Monitor $=6$ \\
\hline Phase 3 & 18 (No) & 0 & 18 & Independent $=10 ;$ Sponsor $=3 ;$ Local $=0 ;$ PI $/$ Medical Monitor $=5$ \\
\hline Phase 3 Total & 34 & 0 & 34 & $\begin{array}{l}\text { Independent = } 18(53 \%) \text {; Sponsor = } 5(15 \%) \text {; Local = 0; PI/Medical } \\
\text { Monitor = } 11(32 \%)\end{array}$ \\
\hline Total & 67 & 0 & 67 & $\begin{array}{l}\text { Independent = } 41(61 \%) \text {; Sponsor = } 8(12 \%) \text {; Local = 0; PI/Medical } \\
\text { Monitor = } 18(27 \%)\end{array}$ \\
\hline
\end{tabular}

For Phase 1 studies the distribution included the following: independent DSMB 65\%, sponsor based DSMB 5\% and no DSMB (PI and/or medical monitor) $30 \%$.

For Phase 2 studies the distribution included the following: independent DSMB 76\%, sponsor based DSMB 15\% and no DSMB (PI and/or medical monitor) $19 \%$.

For phase 3 studies the distribution included the following: independent DSMB (53\%), sponsor based DSMB (15\%) and no DSMB (PI and/or medical monitor) (32\%).

The overall distribution across all studies included the following: independent DSMB 61\%, sponsor based DSMB (12\%) and no DSMB (PI and/or medical monitor) (27\%).

A comparison of blinded vs. unblinded studies across all study phases was also examined Table 2 .
The results showed no difference between blinded and unblinded studies in terms of distribution of DSMB patterns with about $60 \%$ being independent for both, about $10 \%$ being sponsor based and about $27 \%$ being PI/medical monitor safety assessments.

Table 2: DSMB pattern analyzed by study blind

\begin{tabular}{ll}
\hline Items & DSMB \\
\hline \multirow{3}{*}{ Blinded all studies (25) } & Independent = 15 (60\%) \\
& Sponsor = $3(12 \%)$ \\
& Local = 0 \\
& PI/Medical Monitor = 7 (28\%) \\
& Independent = 26 (62\%) \\
& Sponsor = 5 (12\%) \\
Unblinded all studies (42) & Local = 0 \\
& PI/Medical Monitor = 11 (26\%) \\
\hline
\end{tabular}


Locally based DSMBs involving PI (other institutional employees) were not utilized in any of these studies. This is in sharp contrast to NIH based clinical trials where local DSMBs play a very prominent role.

\section{Discussion}

The IRB is the major gatekeeper to ensure DSMPs are prepared and filed. The CHLA IRB requires that a DSMP be filed with the initial study application. In the sample set $100 \%$ of the applications had the DSMP template required by the IRB. Furthermore, the information contained in the DSMP required by the IRB was complete and provided essential details regarding whether there was a DSMB, and if there was one, how it was constituted, how frequently it would meet and the decision making criteria for safety assessments. In none of the instances was a sponsor based DSMP communicated to the PI by the sponsor. Indeed review of the correspondence between the IRB and the PI revealed that in many instances the sponsor did not have a prepared DSMP and in several instances was reluctant to provide information about the DSMB except after a formal request to do so by the IRB. The conclusion here is that without IRB intervention there would be no DSMP filed with the study application and data and safety assessment infrastructures would remain obscure.

PI/Medical monitor based safety assessment occurs in a significant portion of pediatric clinical trials. Both $\mathrm{NIH}$ and FDA guidelines suggest that when children are included as subjects a DSMB is recommend for phase I, phase II, and Phase III clinical trials. Despite the congruence of NIH and FDA guidelines, the studies presented here suggest that a significant number $(30 \%)$ of the clinical trials rely on the PI and the medical director to make safety assessments. It was found during this study, that the main mechanism utilized here was the case report form for SAEs which is completed by the PI and mailed to the sponsors' medical safety officer. Whether a collective safety review occurs at the sponsor's end is unclear and data on this was not available. What was somewhat surprising was the large number, (about a third of the clinical trials), that used this mechanism.

Sponsors using DSMBs predominantly use independent DSMBs. Where sponsors use a DSMB, the choice is to use the independent DSMB rather than sponsor based DSMB. This is in agreement with the spirit of the NIH and FDA guidelines. A shortcoming of our study, however is that absolute independence of the DSMB could not be verified. For example, voting and non voting membership, and potential financial conflict of interest with respect to the sponsor on the part of committee members could not be verified. Rather we had to rely on the "global" assertions of independence provided by the sponsor.
The pattern of DSMB usage is similar across study phases and study blinding. A surprising finding was that the relative distribution of DSMB usage remained essentially the same across phase I, II and III clinical trials. Also very little variation was noted in blinded $v s$. non-blinded trials. It was anticipated that in accordance with FDA and NIH recommendations the pattern would heavily favor the use of DSMBs in phase III trials over phase I and II trials and that independent DSMBs use would predominate in blinded over unblinded trials. Such a difference in DSMB pattern usage was not observed in our study.

Local DSMCs are not used by sponsors in clinical trials. The local DSMCs are comprised of the PI and fellow staff members generally derived from the same department with an institutional biostatistician involved in carrying out the quantitative analysis. Such an arrangement was not observed in any of the studies reviewed. This is in sharp contrast to NIH based clinical trials with NIH institute sponsorship where such a mechanism is frequently utilized.

\section{Conclusion}

Our results indicate that despite the mandate by both the NIH and the FDA that pediatric clinical trials should have a DSMB, several sponsored clinical trials seem not to comply with this guidance. Instead a medial monitor and in some instances the principal investigator is used instead of the DSMB. This has several limitations:

(1) In a multi site trial assessment of adverse events at a single site cannot provide meaningful insights into the overall safety profile of the trial. Statistical analysis of multi-site data is essential and cannot be accomplished by a single site reporting of the medical monitor or the principal investigator.

(2) The second limitation concerns the timelines of applying stopping rules if adverse events of sufficient seriousness are indeed observed. A biostatistician is clearly required for such a sophisticated analysis and indeed some sponsors may opt for an independent biostatistical analysis from an individual unaffiliated with the sponsor.

(3) The fact that DSMPs are not submitted routinely by sponsors makes the role of IRB all the more important in ensuring that a DSMP is submitted in a timely fashion at the time of the initial IRB review.

(4) Finally the role of quality assurance should be emphasized. Where variations are observed in DSMB practices it is important that the IRB follow a set of conservative guidelines in a consistent manner. Review of the IRB reviewing practices regarding DSMBs can be useful in ensuring fair and consistent reviews of sponsored clinical trials. 


\section{References}

[1] Gordon V, Sugarman J, Kass, N. The interface of Data safety monitoring board and insutional review boards in Randomized clinical trials. IRB: Ethics and Human Research. 1998; 20(1): 1-5. PMid: 11655324. http://dx.doi.org/10.2307/3564020

[2] Cairns, JA, Hallstrom, A, Held, P. Should all trials have a Data and Safety Monitoring Committee. American Heart Journal. 2001; 141(4): 156-163. PMid: 11136502. http://dx.doi.org/10.10 $67 / \mathrm{mhj} .2001 .112501$

[3] Dixon, D, Freedman, R, Herson, J, et al. Guidelines for data and safety monitoring for clinical trials not requreing traditional data monitoring committees. Clinical Trials. 2006; 3: 314-319. PMid: 16895048. http://dx.doi.org/10.1191/1740774506c n149oa
[4] Sydes, MR, Spiegelhalter, DJ, Altman, D, et al. Systematic qualitative review of the literature on data monitoring committees for randomized controlled trials. Clinical Trials. 2004; 1: 60-79. PMid: 16281463. http://dx.doi.org/10.1191/1740774504c n004rr

[5] Armstrong, PW, Furberg, C. Clinical trial data and data and safety monitoring boards; the search for a constitution. Circulation. 1995; 91: 901-904. PMid: 7828320. http://dx.doi.org/10.1161/0 1. CIR. 91.3.901

[6] Morse, MA, Califf, RM, Sugarman, J. Monitoring and Ensuring Safety during clinical research. JAMA. 2001; 285(9): 1201-1205. PMid: 11231751. http://dx.doi.org/10.1001/jama.285.9. 1201

[7] Fisher, L, Kilbaner, M. Regulatory Issues for Data and Safety monotring Committees. American Heart Journal. 2001; 141(4): 536-541. PMid: 11275916. http://dx.doi.org/10.1067/mhj .2001 .113839 